\title{
Tea Polyphenols Selectively Inhibit Proliferation and Migration of Airway Smooth Muscle Cells
}

\author{
Mengmeng Yang (First author) \\ School of Pharmaceutical Engineering and Life Science, Changzhou University, \\ Changzhou, China, 213164 \\ Fang Liang (First author) \\ Department of Light Industry Engineering, Changzhou Vocational Institute of Light \\ Industry, Changzhou, China, 213164 \\ Defeng Xu (Corresponding author) \& Yue Wang \\ School of Pharmaceutical Engineering and Life Science, Changzhou University, \\ Changzhou, China, 213164
}

Huan Zhou (Corresponding author)

School of Mechanical Engineering, Jiangsu University of Technology, Changzhou, China, 213001

\section{Darcy E Wagner}

Department of Experimental Medical Sciences, Lund University, Lund, Sweden, 22184

Lei Liu

Institute of Biomedical Engineering and Health Sciences, Changzhou University Changzhou, China, 213164

Received: March 19, 2018 Accepted: April 2, 2018

doi:10.5296/jbls.v9i2.13018 URL: https://doi.org/10.5296/jbls.v9i2.13018 


\section{Abstract}

Airway smooth muscle cells (ASMCs) have been recommended as a target for the treatment of inflammation and narrowing of the airways in asthma. New safe and efficient approaches to relieve symptoms caused by ASMCs is highly desired. Inspired by the inhibitory effects of tea polyphenols on vascular smooth muscle cells (VSMCs), tea polyphenols were applied in the current work to evaluate their regulation of ASMCs in vitro. A dose-dependent decrease of ASMCs density was observed after $24 \mathrm{~h}$ incubation with tea polyphenols. Additionally, ASMCs were significantly more sensitive to tea polyphenols than human bronchial epithelial cells (HBECs). Tea polyphenol treatment led to a dose dependent inhibition on ASMC migration and reduced the gene expression of nuclear factor- $\kappa \mathrm{B}(\mathrm{NF}-\kappa \mathrm{B})$. In studies which compared the four main polyphenolic constituents of tea polyphenols-including epicatechin (EC), epicatechingallate (ECG), epigallocatechin (EGC) and epigallocatechingallate (EGCG) - on the proliferation of ASMCs, EGC was identified as being the most potent. These results suggest tea polyphenols are a promising agent for ASMCs targeted asthma control.

Keywords: Tea polyphenols; asthma; airway smooth muscle cells; antioxidant; EGC

\section{Introduction}

The acute narrowing of the airway and airflow obstruction in asthma is caused by abnormal contractions as well as mass increase of airway smooth muscle (ASM) (Solway \& Irvin, 2007). Airway smooth muscle cells (ASMCs) are known to release a wide range of cytokines such as interleukin-5 (IL-5), interleukin-13 (IL-13), and transforming growth factor- $\beta 1$ (TGF- $\beta 1$ ) which cause inflammation in asthma (Doeing \& Solway, 2013). Therefore ASMCs has been recommended as an important therapeutic target for asthma treatment. Several drugs targeting ASMCs have been developed over the past few decades, including $\beta$-agonists, anti-histamines and anti-leukotrienes (Siddiqui et al., 2013). Unfortunately, while they can relieve the symptoms of dyspnea, they have limited effects on the structural alterations of the airways caused by diseased ASM.

Bronchial thermoplasty is an FDA approved technology to control increased ASM mass in severe asthmatics which do not respond to conventional therapies such as inhaled corticosteroids (ICS) and long-acting $\beta 2$-agonists (LABAs) (Wahidi \& Kraft, 2012). It aims to reduce ASM mass in the airways via delivering controlled thermal energy through a bronchoscopically placed probe to the airway walls within a series of three bronchoscopies. Improvements in the patient's quality of life have been confirmed after bronchial thermoplasty (Wechsler et al., 2013). Unfortunately, this technology is only applicable in bronchi with diameters larger than $3 \mathrm{~mm}$ and involves considerable complications (three separate bronchoscopic procedures, potential damage to healthy tissue, high cost, etc.) (Solway \& Irvin, 2007). Therefore, bronchial thermoplasty has thus far only been recommended for severe asthma patients and further refinement is needed to improve its performance and potential application to a broader patient population. Development of safe and efficient approaches to control or reduce ASM mass beyond bronchial thermoplasty is needed. 


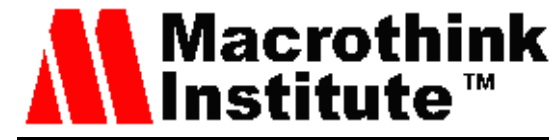

Tea polyphenols are natural plant compounds found in tea, including catechins, theaflavins, tannins, and flavonoids. These chemicals are speculated to provide potential health benefits to human beings. Tea polyphenols have been previously found to inhibit the adhesion, migration and proliferation of vascular smooth muscle cells (VSMCs). The exposure of tea polyphenols to VSMCs can suppress the level of phosphorylated Akt (p-Akt), nuclear factor- $\kappa \mathrm{B}(\mathrm{NF}-\kappa \mathrm{B})$, tumor suppressor p53 and each subsequent target genes or proteins (Hofmann \& Sonenshein, 2003; Yallapu, Jaggi, \& Chauhan, 2010). However, despite the known effects of tea polyphenols on VSCMCs, there is no information available on their effects on ASMCs. Therefore, we hypothesized that tea polyphenols can be used as a potential agent to control or reduce the mass of ASM.

\section{Materials and Methods}

2.1 Effects of tea polyphenols on the proliferation of ASMCs and human bronchial epithelial cells (HBECs)

ASMCs were derived from rat by our group as previously described (Wang et al., 2016) and HBECs were purchased from ATCC. Cells for all studies were used between passage 3 and 5 . Reagents unless stated otherwise used for in vitro study were from Thermo Fisher Scientific, Inc. (Waltham, MA, USA). The tea polyphenols tested (98\% purity) were purchased from Yuanye Co., Ltd (Shanghai, China).

In the proliferation study, ASMCs were trypsinized and seeded at $1 \times 10^{4}$ cells/well in $96-$ well plates with Dulbecco's modified Eagle's medium (DMEM) contained $0.5 \%$ fetal bovine serum (FBS). After $12 \mathrm{~h}$ incubation at $37^{\circ} \mathrm{C}$ under humidified atmosphere of $5 \% \mathrm{CO}_{2}$, ASMCs were cultured with different concentrations of tea polyphenols $(0,0.01,0.025,0.05,0.075$, $0.1 \mathrm{mg} / \mathrm{mL}, \mathrm{n}=6$ ) for $24 \mathrm{~h}$. Cell proliferation was assessed by MTT assay. The cellular morphology of treated ASMCs were visualized using an optical microscope (Carl Zeiss). HBECs were seeded at $1 \times 10^{4}$ cells/well and treated similarly to ASMCs.

\subsection{Migration assay of ASMCs exposed to tea polyphenols}

ASMCs were seeded at $1 \times 10^{5}$ cells/well in 6-well plates and incubated at $37^{\circ} \mathrm{C}$ and $5 \% \mathrm{CO}_{2}$ with complete medium until $90 \%$ confluence was reached. After that, a horizontal scratch wound was generated across each well using a pipette tip to make the cell free area with clear edges. The residual cells were washed twice with PBS and then treated with different concentrations of tea polyphenols $(0,2.5,5,10 \mu \mathrm{g} / \mathrm{mL})$. The migration behaviors of ASMCs were characterized using live cell imaging system (Carl Zeiss) after 0, 24, and $48 \mathrm{~h}$ of incubation.

\subsection{RT-PCR study of NF- $\kappa B$ expression in tea polyphenols treated ASMCs}

In brief, $5 \times 10^{4}$ ASMCs were added to each well of a 6-well plate and grown in DMEM. Cells were monitored until they reached approximately $80 \%$ confluence, the culture medium was then removed and DMEM with $0,5,10 \mu \mathrm{g} / \mathrm{mL}$ tea polyphenols was added. After $24 \mathrm{~h}$ incubation, the total RNA was extracted with Trizol and reverse transcribed to cDNA using random primers. Subsequently, the expression level of $\mathrm{NF}-\kappa \mathrm{B}$ was analyzed by real time 


\section{Al Macrothink}

polymerase chain reaction (RT-PCR) using the real-time fluorescence quantitative PCR system (Applied Biosystems) with the following primer pairs:

NF-кB:Forward5'-TTATCAACCACCCCAACGGG-3',

\section{Reverse5'-GCTCCTGAGCGTTGACTTCT-3';}

GAPDH:Forward5'-GCCATCAACGACCCCTTCAT-3',

\section{Reverse5'-GATGGTGATGGGTTTCCCGT-3'.}

\subsection{The effects of EC, ECG, EGC, EGCG or Vitamin C on the proliferation of ASMCs}

It is well-known that tea polyphenols are a complex of different polyphenols. Four of the main polyphenolic constituents of tea polyphenols including epicatechin (EC), epicatechingallate (ECG), epigallocatechin (EGC), epigallocatechingallate (EGCG) were applied to evaluate their ability to inhibit the proliferation of ASMCs (Table 1). All of the selected compounds belong to catechins, which occupy over $70 \%$ polyphenolic constituents of tea polyphenols. All chemicals had a purity of $98 \%$ and were purchased from Nanjing Spring \& Autumn Biological Engineering Co., Ltd. The in vitro experiment procedures followed section 2.1 above and used a polyphenol concentration of $10 \mu \mathrm{g} / \mathrm{mL}$. In addition, EGC was further applied to ASMCs with a concentration ranging from 1 to $20 \mu \mathrm{g} / \mathrm{mL}$.

Table 1 Molecular structure of tested tea polyphenols



Vitamin C, a known anti-oxidant, was used to investigate whether the effect of the tea polyphenols is based on its anti-oxidative properties (Lo, Hung, Huang, \& Wu, 2007). Experiments which included vitamin $\mathrm{C}$ followed the same procedure as described in section 2.1 using $10 \mu \mathrm{g} / \mathrm{mL}$ of vitamin C (Aladdin, Shanghai, China) instead of tea polyphenols and EGCG was used for comparisons. 


\section{Macrothink}

\subsection{Statistical analysis}

All experiments were independently repeated at least three times. Statistical analysis for multiple comparisons was performed using t-test. A level of $p<0.05$ was considered statistically significant.

\section{Results}

\subsection{Effects of tea polyphenols on the proliferation of ASMCs and HBECs}

ASMCs exposed to tea polyphenols (Fig.1a) exhibited a dose-dependent decrease in metabolic activity after $24 \mathrm{~h}$ incubation as assessed by MTT $(\mathrm{p}<0.05)$. Representative images of ASMCs treated with the tea polyphenols are shown in Fig.1b-c. The morphology of ASMCs qualitatively changed from spindle-shaped to irregular when high doses of tea polyphenols were applied. In order to determine if tea polyphenols also effect other cell types in the airways, we repeated these same experiments using HBECs. In contrast to the dose-dependent effects we observed in ASMCs, we observed no significant decreases in HBECs with tea polyphenol treatment, even in the presence of increasing doses (Fig.1d). In order to better understand the kinetics of the tea polyphenols in inhibiting cellular viability, we determined the half maximal inhibitory concentration (IC50) value of tea polyphenols on the ASMCs and HBECs. We calculated these to be 14.59 and $4612 \mu \mathrm{g} / \mathrm{mL}$ respectively, significantly different from each other (Fig.2). Therefore, in all subsequent studies, we used tea polyphenols at a maximum concentration of $10 \mu \mathrm{g} / \mathrm{mL}$.


Fig.1 Tea polyphenols selectively reduce the proliferation of ASMCs: (a) proliferation of ASMCs after exposure to different concentrations of tea polyphenols for $24 \mathrm{~h}$, “*” refers to p<0.05; (b) morphology of normal ASMCs under optical scope; (c) morphology of ASMCs treated with $100 \mu \mathrm{g} / \mathrm{mL}$ tea polyphenols; (d) proliferation of HBECs after exposed to different concentrations of tea polyphenols for $24 \mathrm{~h}$ 


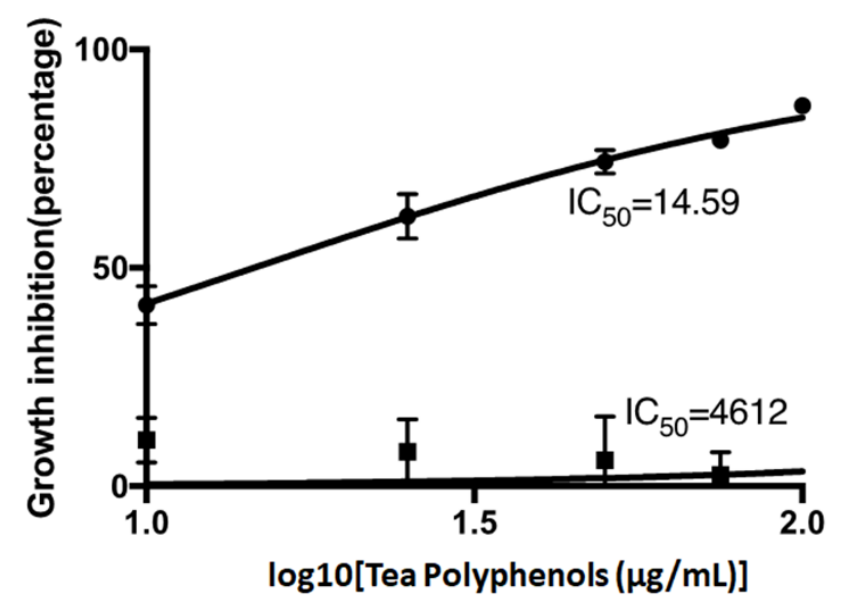

Fig.2 $\mathrm{IC}_{50}$ values of tea polyphenols on the ASMCs (circles) and HBECs (squares)

\subsection{Inhibitory effects of tea polyphenols on migration of ASMCs}

The inhibitory effects of tea polyphenols on migration of ASMCs were investigated by performing in vitro migration assays where a small scrape was made across a confluent monolayer of ASMCs. It could be seen visually that there was complete recovery of the denuded space by the migration and proliferation of ASMCs at $48 \mathrm{~h}$ (Fig.3a). On the contrary, ASMCs exposed tea polyphenols had statistically significant delays $(\mathrm{p}<0.05)$ in closure of the denuded space at each time point; the level of delay increased with the increased tea polyphenols concentrations (Fig.3). When the concentration of tea polyphenols reached 10 $\mu \mathrm{g} / \mathrm{mL}$, almost no cell migration or proliferation was observed.


Fig.3 Tea polyphenols impact migration in a dose dependent manner. Migration of ASMCs after exposure to different concentrations of tea polyphenols: (a) tea polyphenols inhibited ASMCs migration in wound healing assay; (b) Quantification of wound closure, higher tea polyphenols concentration resulted in less wound closure. Scale bar is $500 \mu \mathrm{m}$ and "*" refers to $\mathrm{p}<0.05$ 


\section{Macrothink}

\subsection{Effects of tea polyphenols on $N F-\kappa B$ expression in ASMCs}

Owing to previous reports which have documented that tea polyphenols can inhibit inflammation, we next explored whether there was a relationship between the concentrations of tea polyphenols with the well-known inflammatory factor, NF- $\kappa B$, in ASMCs. When tea polyphenols concentration of $5 \mu \mathrm{g} / \mathrm{mL}$ was applied to ASMCs, NF- $\kappa B$ expression did not significantly different as compared to control group. However, when concentrations of 10 $\mu \mathrm{g} / \mathrm{mL}$ was used, we observed a strong inhibition of NF- $\kappa \mathrm{B}$ expression in ASMCs $(\mathrm{p}<0.05)$ (Fig 4).

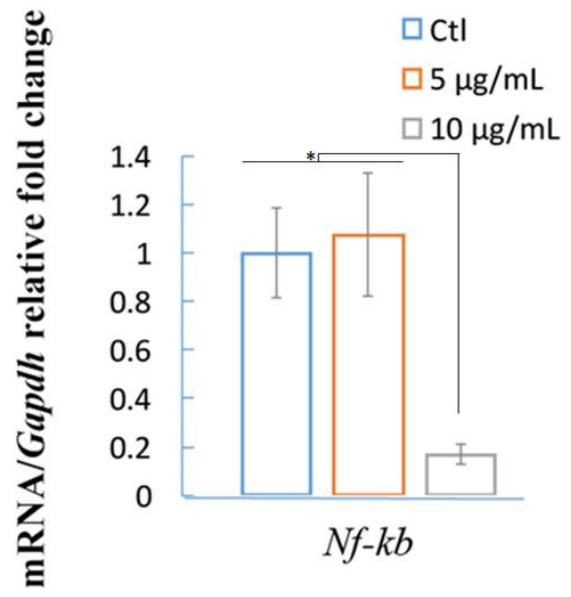

Fig.4 NF-KB mRNA expression was inhibited by tea polyphenols treatment at high dosage in ASMC, “*” refers to $\mathrm{p}<0.05$

\subsection{The effects of EC, ECG, EGC, EGCG, and Vitamin C on the proliferation of ASMCs}

In order to determine which of the main constituents of tea polyphenols were responsible for the observed effects, we next treated with each of the four main components: EC, ECG, EGC, and EGCG. We found that all four tested polyphenols EC, ECG, EGC, EGCG all significantly reduced the proliferation of ASMCs ( $\mathrm{p}<0.05)$ (Fig. 5a). Because EGC was observed to be the strongest inhibitor of ASMCs, we then sought to further understand the dose dependent effects of EGC on ASMCs behavior. We found that EGC treatment resulted in a dose-dependent decrease of ASMCs (Fig. 5b).

Considering the fact tea polyphenols are well known anti-oxidants, we sought to determine if the effects which we observed were due to the anti-oxidant behavior of tea polyphenols. In order to address this question, we treated ASMCs with the well-known anti-oxidant Vitamin C. While Vitamin C at a concentration of $10 \mu \mathrm{g} / \mathrm{mL}$ inhibited the proliferation of ASMCs $(\mathrm{p}<0.05)$, this inhibition was not to the same extent at which EGCG effected proliferation at the same concentration (Fig.6), indicating that the effect of the tea polyphenols is not exclusively due to its anti-oxidative properties. 



Fig.5 In vitro results of the proliferation of ASMCs after exposure to polyphenolic constituents of tea polyphenols: (a) EC, ECG, EGC, EGCG all inhibited the proliferation of ASMCs; (b) EGC showed a dose-dependent inhibitory effect on ASMCs proliferation; " “” refers to $\mathrm{p}<0.05$

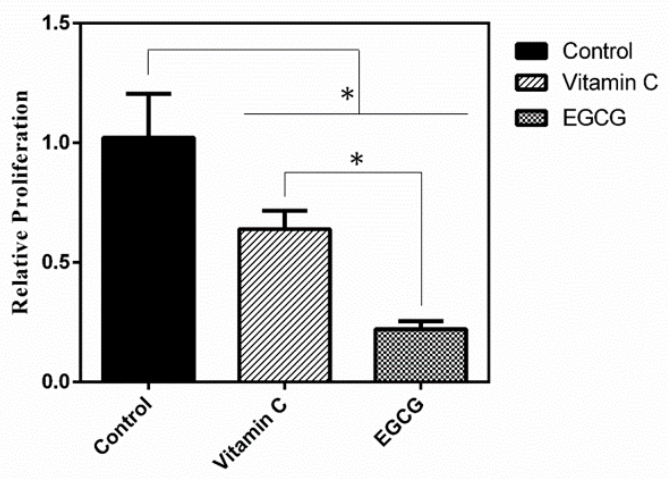

Fig.6 Vitamin C inhibited the proliferation of ASMCs ( $<<0.05)$, but not as well as EGCG of the same concentration $(\mathrm{p}<0.05)$

\section{Discussion}

Tea is the one of the most frequently consumed beverages worldwide, and its significant anti-oxidative, anti-inflammatory, anti-microbial, anti-carcinogenic, anti-hypertensive, neuro-protective, cholesterol-lowering, and thermogenic properties are well documented (Hayat, Iqbal, Malik, Bilal, \& Mushtaq, 2015). With regard to their potential in asthma therapies, the beneficial effects of tea polyphenols have been previously illustrated. Hirota et al. reported that tea consumption exerted an inhibitory effect on airway hyperresponsiveness in mice and attenuated increases in allergic inflammation and increased the production of adiponectin (Hirota, Ngatu, Miyamura, Nakamura, \& Suganuma, 2011). Reinhart et al. observed that the polyphenolic constituent EGCG can reduce airway inflammation in mice by binding to proinflammatory chemokines and inhibiting inflammatory cell recruitment (Qin et al., 2011). Additionally, tea polyphenols can also provide protection for HBECs exposed to oxidative damage (Qing, Chen, \& Xiang, 2010). Beyond these impressive results of tea polyphenols, to the best of our knowledge this is the first report which explores their 
inhibitory effects on ASMCs. As described in introduction, the inhibitory effect of tea polyphenols on another smooth muscle cells VSMCs are well studied. Meanwhile, though VSMCs and ASMCs are both considered as smooth muscle cells, they are still quite different from each other (Fernandes, McConville, Stewart, Kalinichenko, \& Solway, 2004): 1) contractile protein, transcription factor expression and regulation of contractile protein promoter activity of ASMCs and VSMCs are different: 2) the embryonic developmental signals in mice required for differentiation of ASM versus pulmonary VSM are distinct; c) in addition, application of ASM-specific gene therapies would induce minimal side effects on other lung smooth muscle tissues. Therefore, whether tea polyphenols can be used as a dietary supplement or drug candidate to control or reduce the mass of ASM instead of VSM was uncertain before this work.

In the present work, our results demonstrated the remarkable effects of tea polyphenols on the proliferation and migration of ASMCs. The proliferation and migration of ASMCs are associated with the growth of airway smooth muscle mass. The proliferation and migration of ASMCs following tea polyphenols treatment showed a significant dose-dependent inhibition, indicating tea polyphenols may be effective in controlling remodeling of ASM. Further, we examined whether tea polyphenols additionally have anti-inflammatory effects. We determined that tea polyphenols can inhibit NF- $\kappa \mathrm{B}$ expression in ASMCs. NF- $\kappa \mathrm{B}$ plays important roles in the pathogenesis of inflammation after asthma occurrence. NF- $\kappa \mathrm{B}$ is a transcription factor expressed in numerous cell types, which plays a key role in the expression of many pro-inflammatory genes; there is a wealth of evidences indicating enhanced NF- $\mathrm{BB}$ pathway activation in asthmatic tissues and much of the underlying inflammation of asthma independent of stimuli, is mediated at least in part, by $\mathrm{NF}-\kappa \mathrm{B}$ mediated signaling events in several cell types (Edwards et al., 2009). Consequently, NF- $\mathrm{B}$ has been recommended as a potential target for the effective treatment for asthma. Our results are the first to demonstrate that tea polyphenols can inhibit NF- $\mathrm{kB}$ in ASMCs though the inhibition of NF-kB could be caused by a cytotoxic effects, but previous studies, have reported on the ability of tea polyphenols to inhibit the activation of NF- $\kappa \mathrm{B}$ in other cell types (Li et al., 2014; Nomura, Ma, Chen, Bode, \& Dong, 2000; Park, Jeong, Jeong, Park, \& Kim, 2011). In summary, these observations point to the potential development of a safe and efficient approach to control or reduce ASMCs caused risks of asthma patients in daily life as an alternative to known asthma control approaches.

A major open question following our study is how tea polyphenols exert the effects we observed. Future studies will be needed to further understand the exact mechanism of action. An intriguing observation in our study is that ASMCs seem to be specifically responsive to tea polyphenols as compared to HBECs. One possible explanation is that specific intracellular pathways which can respond to tea polyphenols only exist in ASMCs, but not in HBECs. This will need to be further clarified in future studies.

In this study, we also evaluated the effects of antioxidant vitamin $\mathrm{C}$ on the proliferation of ASMCs. An inhibitory effect was also observed, though not as significant as EGCG. Vitamin $\mathrm{C}$ has been well accepted as a highly effective anti-oxidant, similar to tea polyphenols. Similarly, in work conducted by Quet al., they reported the ability of another well-known 
antioxidant, curcumin, to inhibit the proliferation of ASMCs in vitro and in vivo (Zeng et al., 2013). Curcumin also belongs to the family of polyphenol, but shows poor water solubility as compared to tea polyphenols. The possible inhibitory mechanisms of curcumin on ASMCs in that study was suggested to be the up-regulation of the expression of caveolin-1 and inhibition of the ERK pathway. These will be important areas of investigation in future work.

Effective anti-oxidants, such as polyphenols, could therefore have potential applications in the control of the excessive growth of ASMCs. Previously, the anti-oxidation ability of EC, ECG, EGC, and EGCG has been shown to be EGCG=EGC>ECG>EC (Choi \& Jung, 2013). However, in current work, EGC was the best inhibitor of ASMCs, indicating that the molecular structure of the specific polyphenol may also have impacts on its effects on ASMCs, although the kinetics have not been fully examined here. Indeed in a previous study of VSMCs, the inhibitory effect of tea polyphenols was suggested to be related to its molecular structure with abundant hydroxyl groups and gallate rings, which possibly bind to specific sites and thus interrupt the exogenous signals required for the proliferation and growth of cells (Han et al., 2006; Kim \& Moon, 2005). Therefore, more detailed studies on the mechanism to guide the design of potent polyphenolic drugs to inhibit ASMCs are needed in future.

\section{Conclusions}

In conclusion, the principal findings of the present work are as follows: 1) tea polyphenols can inhibit the proliferation, migration and $\mathrm{NF}-\kappa \mathrm{B}$ expression of ASMCs; 2) the effects of tea polyphenols on ASMCs may be partly associated with its anti-oxidative property; 3) its polyphenolic constituents with different molecular structure exhibit variation on the inhibitory effect of ASMCs proliferation, and EGC seems to be the best candidate among tested catechins. Our observations may have both dietary and therapeutic implications in the treatment of asthma. Tea polyphenols may be a safe and efficient approach to control or reduce ASM mass.

\section{Acknowledgements}

This work was partially supported by Research Innovation Program for College Graduates of Jiangsu Province (No.SJCX17_0709), The General Program of Natural Science Foundation of Jiangsu Province (No. BK20171201), International Science and Technology Cooperation Program of Changzhou (No. CZ210170001), and National Natural Science Foundation of China (No. 11532003).

Mengmeng Yang \& Fang Liang contributed equally to this work.

\section{References}

Choi, D. S., \& Jung, M. Y. (2013). Protective Activities of Catechins on Singlet Oxygen Induced Photooxidation of alpha-Terpinene in Methanol: Structure and Singlet Oxygen Quenching Activity Relationship. Food Science and Biotechnology, 22(1), 249-256. doi:10.1007/s10068-013-0034-8

Doeing, D. C., \& Solway, J. (2013). Airway smooth muscle in the pathophysiology and 
treatment of asthma. Journal of Applied Physiology, 114(7), 834-843.

doi:10.1152/japplphysiol.00950.2012

Edwards, M. R., Bartlett, N. W., Clarke, D., Birrell, M., Belvisi, M., \& Johnston, S. L. (2009). Targeting the NF- $\kappa \mathrm{B}$ pathway in asthma and chronic obstructive pulmonary disease. Pharmacology \& Therapeutics, 121(1), 1-13.

doi:http://dx.doi.org/10.1016/j.pharmthera.2008.09.003

Fernandes, D. J., McConville, J. F., Stewart, A. G., Kalinichenko, V., \& Solway, J. (2004). Can we differentiate between airway and vascular smooth muscle? Clinical and Experimental Pharmacology and Physiology, 31(11), 805-810. doi:10.1111/j.1440-1681.2004.04084.x

Han, D. W., Lim, H. R., Baek, H. S., Lee, M. H., Lee, S. J., Hyon, S. H., \& Park, J. C. (2006). Inhibitory effects of epigallocatechin-3-O-gallate on serum-stimulated rat aortic smooth muscle cells via nuclear factor-kappa B down-modulation. Biochemical and Biophysical Research Communications, 345(1), 148-155. doi:10.1016/j.bbrc.2006.04.072

Hayat, K., Iqbal, H., Malik, U., Bilal, U., \& Mushtaq, S. (2015). Tea and Its Consumption: Benefits and Risks. Critical Reviews in Food Science and Nutrition, 55(7), 939-954.

doi:10.1080/10408398.2012.678949

Hirota, R., Ngatu, N. R., Miyamura, M., Nakamura, H., \& Suganuma, N. (2011). Goishi tea consumption inhibits airway hyperresponsiveness in BALB/c mice. Bmc Immunology, 12. doi:10.1186/1471-2172-12-45

Hofmann, C. S., \& Sonenshein, G. E. (2003). Green tea polyphenol epigallocatechin-3 gallate induces apoptosis of proliferating vascular smooth muscle cells via activation of p53. Faseb Journal, 17(2), 702-+. doi:10.1096/fj.02-0665fje

Kim, C.-H., \& Moon, S.-K. (2005). Epigallocatechin-3-gallate causes the p21/WAF1-mediated G1-phase arrest of cell cycle and inhibits matrix metalloproteinase-9 expression in TNF- $\alpha$-induced vascular smooth muscle cells. Archives of Biochemistry and Biophysics, 435(2), 264-272. doi:http://dx.doi.org/10.1016/j.abb.2004.12.022

Li, Y. W., Zhang, Y., Zhang, L., Li, X., Yu, J. B., Zhang, H. T., . . . Liu, H. G. (2014). Protective effect of tea polyphenols on renal ischemia/reperfusion injury via suppressing the activation of TLR4/NF-kappa B p65 signal pathway. Gene, 542(1), 46-51.

doi:10.1016/j.gene.2014.03.021

Lo, H.-M., Hung, C.-F., Huang, Y.-Y., \& Wu, W.-B. (2007). Tea polyphenols inhibit rat vascular smooth muscle cell adhesion and migration on collagen and laminin via interference with cell-ECM interaction. Journal of Biomedical Science, 14(5), 637-645.

doi:10.1007/s11373-007-9170-6

Nomura, M., Ma, W. Y., Chen, N. Y., Bode, A. M., \& Dong, Z. G. (2000). Inhibition of 12-O-tetradecanoylphorbol-13-acetate-induced NF-kappa B activation by tea polyphenols, (-)-epigallocatechin gallate and theaflavins. Carcinogenesis, 21(10), 1885-1890. doi:10.1093/carcin/21.10.1885 


\section{MInstitute Macrothink $^{\text {Int }}$}

Park, S. J., Jeong, J. M., Jeong, H.-S., Park, J.-S., \& Kim, N.-H. (2011). Effects of Epigallocatechin-3-Gallate on the Expression of TGF-beta1, PKC alpha/betaII, and NF-kappaB in High-Glucose-Stimulated Glomerular Epithelial Cells. Chonnam medical journal, 47(2), 116-121. doi:10.4068/cmj.2011.47.2.116

Qin, S. L., Alcorn, J. F., Craigo, J. K., Tjoeng, C., Tarwater, P. M., Kolls, J. K., \& Reinhart, T. A. (2011). Epigallocatechin-3-Gallate Reduces Airway Inflammation in Mice through Binding to Proinflammatory Chemokines and Inhibiting Inflammatory Cell Recruitment. Journal of Immunology, 186(6), 3693-3700. doi:10.4049/jimmunol.1002876

Qing, C., Chen, P., \& Xiang, X. (2010). Effect of tea polyphenols on oxidative damage and apoptosis in human bronchial epithelial cells induced by low-dose cigarette smoke condensate. Zhong nan da xue xue bao. Yi xue ban = Journal of Central South University. Medical sciences, 35(2), 123-128. doi:10.3969/j.issn.1672-7347.2010.02.006

Siddiqui, S., Redhu, N. S., Ojo, O. O., Liu, B., Irechukwu, N., Billington, C., . . Moir, L. M. (2013). Emerging airway smooth muscle targets to treat asthma. Pulmonary Pharmacology \& Therapeutics, 26(1), 132-144. doi:10.1016/j.pupt.2012.08.008

Solway, J., \& Irvin, C. G. (2007). Airway smooth muscle as a target for asthma therapy. New England Journal of Medicine, 356(13), 1367-1369. doi:10.1056/NEJMe078005

Wahidi, M. M., \& Kraft, M. (2012). Bronchial Thermoplasty for Severe Asthma. American Journal of Respiratory and Critical Care Medicine, 185(7), 709-714.

doi:10.1164/rccm.201105-0883CI

Wang, Y., Lu, Y., Luo, M., Shi, X., Pan, Y., Zeng, H., \& Deng, L. (2016). Evaluation of pharmacological relaxation effect of the natural product naringin on in vitro cultured airway smooth muscle cells and in vivo ovalbumin-induced asthma Balb/c mice. Biomedical Reports, $5,715-722$.

Wechsler, M. E., Laviolette, M., Rubin, A. S., Fiterman, J., Lapa e Silva, J. R., Shah, P. L., . . . Castro, M. (2013). Bronchial thermoplasty: Long-term safety and effectiveness in patients with severe persistent asthma. Journal of Allergy and Clinical Immunology, 132(6), 1295-1302.e1293. doi:https://doi.org/10.1016/j.jaci.2013.08.009

Yallapu, M. M., Jaggi, M., \& Chauhan, S. C. (2010). Beta-Cyclodextrin-curcumin self-assembly enhances curcumin delivery inprostate cancer cells. Colloids Surface B, 79. doi:10.1016/j.colsurfb.2010.03.039

Zeng, X., Cheng, Y., Qu, Y. J., Xu, J. D., Han, Z. Y., \& Zhang, T. T. (2013). Curcumin inhibits the proliferation of airway smooth muscle cells in vitro and in vivo. International Journal of Molecular Medicine, 32(3), 629-636. doi:10.3892/ijmm.2013.1425 


\section{Copyright Disclaimer}

Copyright for this article is retained by the author(s), with first publication rights granted to the journal.

This is an open-access article distributed under the terms and conditions of the Creative Commons Attribution license (http://creativecommons.org/licenses/by/3.0/). 